Annuaire du Collège de France 2017-2018

\title{
Atomes et rayonnement
}

Jean Dalibard

\section{OpenEdition}

Journals

Édition électronique

URL : https://journals.openedition.org/annuaire-cdf/15463

DOI : 10.4000/annuaire-cdf.15463

ISBN : 978-2-7226-0572-5

ISSN : 2109-9227

Éditeur

Collège de France

Édition imprimée

Date de publication : 30 décembre 2020

Pagination : 43-50

ISBN : 978-2-7226-0516-9

ISSN : 0069-5580

Référence électronique

Jean Dalibard, « Atomes et rayonnement », L'annuaire du Collège de France [En ligne], 118 | 2020, mis en ligne le 01 avril 2021, consulté le 22 août 2022. URL : http://journals.openedition.org/annuaire-cdf/ 15463 ; DOI : https://doi.org/10.4000/annuaire-cdf.15463 


\title{
ATOMES ET RAYONNEMENT
}

\author{
Jean DALIBARD \\ Membre de l'Institut (Académie des sciences), \\ professeur au Collège de France
}

Mots-clés : matière, topologie, gaz quantiques

La série de cours et séminaires « La matière topologique et son exploration avec les gaz quantiques » est disponible, en audio et/ou en vidéo, sur le site internet du Collège de France (https://www.college-de-france.fr/site/jean-dalibard/course-2017-2018.htm) ainsi que les notes de cours au format pdf (http://www.phys.ens.fr/ dalibard/CdF/2018/ notes_cours_total.pdf).

\section{ENSEIGNEMENT}

COURS ET SÉMINAIRES - LA MATIÈRE TOPOLOGIQUE ET SON EXPLORATION AVEC LES GAZ QUANTIQUES

\section{Introduction}

Comment classifier les états de la matière ? Au-delà des considérations usuelles fondées sur les symétries géométriques, l'application de concepts issus de la topologie conduit actuellement à des développements fascinants. Initialement proposés pour aborder certains problèmes de l'état solide comme l'effet Hall quantique, ces concepts irriguent désormais les domaines de la physique atomique et de l'optique. La matière topologique se caractérise par des propriétés spécifiques robustes, qui subsistent quand on modifie (modérément) les paramètres du système ou quand on lui ajoute du désordre. Les matériaux topologiques peuvent par exemple présenter une conductance quantifiée ou des états de bord se propageant sans pertes ; ils sont ainsi à l'origine de dispositifs nouveaux, notamment en photonique. Le cours de cette année s'est attaché à établir les bases de ce nouveau champ de recherche pour le cas de particules ou d'ondes en mouvement dans un environnement spatialement périodique. Partant de la notion de phase géométrique (ou phase de Berry), nous avons abordé successivement 
des problèmes à une, puis à deux dimensions. Nous avons également illustré nos résultats par une série d'expériences récentes, menées avec des gaz d'atomes froids ou avec des photons.

\section{Cours 1 - De la phase de Berry à la topologie d'une bande d'énergie}

Ce premier cours a été consacré à la mise en place de notions qui jouent un rôle central dans l'ensemble de cet enseignement. La première concerne la phase géométrique, également appelée phase de Berry; elle nous indique comment évolue un système physique quand on varie lentement un paramètre de contrôle; cette notion est particulièrement importante quand le paramètre revient à sa valeur initiale à l'issue de l'évolution. Nous utilisons ici cette notion dans le cadre de la physique quantique, mais elle est également pertinente en physique classique, le pendule de Foucault en constituant une très belle illustration. La deuxième notion importante porte sur le formalisme décrivant les systèmes quantiques « à deux niveaux », c'està-dire des systèmes dont l'espace des états est de dimension 2. Nous avons donc également rappelé quelques éléments importants de la physique du spin $1 / 2$, en particulier la représentation d'un état de ce spin à l'aide de la sphère de Bloch. Pour finir, nous avons résumé les propriétés spécifiques des systèmes périodiques d'espace, en particulier le théorème de Bloch et la notion de bande d'énergie. Nous avons illustré ces propriétés sur un premier exemple de système topologique, le modèle proposé par Su, Schrieffer et Heeger (SSH).

\section{Cours 2 - Topologie à une dimension : du modèle SSH aux modes de Majorana}

Ce cours a été consacré à la recherche de manifestations concrètes de la topologie d'une bande d'énergie dans un problème unidimensionnel. Le point central a porté sur l'existence d'états de bord robustes, apparaissant à l'interface entre deux phases de topologies différentes. Dans un premier temps, nous nous sommes concentrés sur le modèle d'une chaîne SSH dont nous avons approfondi la caractérisation en termes d'enroulement autour de la sphère de Bloch. Nous avons montré l'existence possible d'états de bord robustes, pour un segment fini de la chaîne ou dans une géométrie semi-infinie. Nous avons ensuite abordé la description d'une expérience menée avec de la lumière en 2017 en France, qui a tiré parti des états de bord pour réaliser un laser topologique. Dans la dernière partie du cours, nous avons comparé cette chaîne SSH avec un autre système modèle remarquable, introduit par Alexei Kitaev pour décrire un supraconducteur topologique à une dimension. Nous avons montré que les états de bord ont alors des propriétés très particulières, décrites par des modes de quasi-particules pour lesquelles opérateurs de création et de destruction coïncident. Ces modes sont baptisés modes de Majorana, par analogie avec le formalisme présenté Ettore Majorana pour analyser l'équation de Dirac. L'étude de ces deux modèles nous a donné l'occasion d'aborder l'importance des symétries dans la stabilité des phases topologiques. Il s'agit de la symétrie de sous-réseau pour le modèle SSH, et de la symétrie particule-trou pour le modèle de Kitaev. Tant que le système possède cette symétrie, les phases topologiques trouvées sont robustes : elles ne peuvent se transformer en une phase normale qu'en fermant un gap entre deux bandes d'énergie, ce qui impose une modification radicale des paramètres de l'hamiltonien. 


\section{Cours 3 - Les pompes adiabatiques}

Dans ce cours, nous sommes allés au-delà du modèle SSH pour examiner ce qui se produit quand on perd la symétrie de sous-réseau. Il y a bien sûr de multiples façons de faire cette généralisation; dans un premier temps, nous avons ajouté un nombre minimum d'ingrédients, en restant dans le régime des liaisons fortes et en relâchant uniquement la contrainte d'équivalence entre sites, ce qui nous a conduits au modèle de Rice-Mele. Nous avons ensuite décrit comment implémenter cette classe générale de réseaux 1D bipartites avec des atomes froids piégés dans des ondes lumineuses stationnaires. Nous avons notamment présenté le principe de la mesure de la phase de Zak, faite en 2013 par Atala et al. Nous avons abordé ensuite le concept de pompe adiabatique, qui émerge quand on s'autorise à varier lentement dans le temps les paramètres du réseau, c'est-à-dire les couplages entre sites ou les énergies sur site. Nous avons vu que, pour une variation périodique de ces paramètres et pour une préparation adéquate des particules, le déplacement du centre de masse de ces particules est quantifié. Nous avons décrit une expérience menée au Japon en 2016 qui a mis en évidence cette quantification dans une expérience d'atomes froids. La pompe adiabatique constitue en fait un problème bidimensionnel, avec une dimension d'espace et une dimension de temps, et nous avons montré qu'il est possible d'exprimer la quantification trouvée en termes de l'intégrale d'une courbure de Berry. Nous avons ainsi fait un premier pas dans les problèmes topologiques de dimension supérieure à 1 : les résultats obtenus dans ce cours vont resurgir sous une forme à peine modifiée pour les problèmes véritablement 2D abordés dans la suite.

\section{Cours 4 - Topologie et courbure de Berry dans un réseau 2D}

À partir de ce cours, nous nous sommes tournés vers l'étude de systèmes topologiques à deux dimensions. Cette géométrie bidimensionnelle a joué un rôle majeur dans l'émergence de concepts topologiques en physique, avec la découverte de l'effet Hall quantique pour les gaz d'électrons confinés dans des puits quantiques. Elle permet de caractériser les phases topologiques par leurs propriétés de transport, ce qui n'était pas possible à une dimension; par ailleurs, elle donne naissance à des nombres topologiques (indices de Chern) plus « robustes » que la phase de Zak qui restait dépendante de la paramétrisation choisie pour un problème physique donné. Nous avons considéré une bande d'énergie isolée et nous avons cherché à caractériser la topologie de cette bande. Nous avons apporté deux types de réponse. La première est mathématique et directement inspirée de ce que nous avons compris pour les pompes géométriques, concernant la couverture de la sphère de Bloch. La seconde réponse est physique et porte sur les propriétés de transport que l'on peut attendre pour ces systèmes. Bien entendu, les deux types de réponses conduisent in fine à la même caractérisation. Pour simplifier notre analyse, nous avons étudié majoritairement des systèmes discrets avec des couplages tunnels n'autorisant des sauts qu'entre sites voisins. Cela nous a permis d'utiliser l'approximation des liaisons fortes et de mener presque tous les calculs de manière analytique. Nous avons également recherché les géométries les plus simples permettant l'apparition d'une topologie non triviale. Comme à 1D, cela nous a conduit à considérer un réseau avec deux sites possibles par cellule unité. Ce type de réseau mène naturellement à l'émergence de points de Dirac dans une géométrie bidimensionnelle, et c'est donc par cette notion que nous avons commencé notre étude, avant de passer à la caractérisation de la topologie. 


\section{Cours 5 - Bandes topologiques et états de bord}

Pour le type de réseaux considérés dans ce cours, l'obtention d'un indice de Chern non nul nécessite de briser l'invariance par renversement du temps. Pour un problème sans spin, cela impose de réaliser des coefficients tunnels complexes. Deux voies peuvent être considérées pour cela. L'une consiste à transposer au réseau la géométrie de l'effet Hall, c'est-à-dire appliquer un champ magnétique uniforme sur ce réseau. Ce champ vient briser l'invariance par translation et peut donner naissance à un spectre d'énergie très riche, avec un grand nombre de sous-bandes d'énergie. L'autre voie, proposée par Haldane en 1988, consiste à garder la même cellule unité que le réseau initial, c'est-à-dire un modèle à deux bandes, tout en augmentant (légèrement) le nombre de couplages tunnel. C'est elle que nous avons explorée dans ce cinquième cours. Après avoir posé les principes du modèle de Haldane, nous avons étudié son implémentation avec des atomes froids, menée dans différents laboratoires entre 2012 et 2017. Nous avons ensuite utilisé ce modèle pour expliquer la correspondance cour-bord, c'est-à-dire l'apparition inévitable de canaux de bord à l'interface entre deux zones de topologie différentes. Nous avons terminé ce cours par la description de la mise en évidence expérimentale de ces canaux de bord sur des systèmes photoniques, publiée au début de l'année 2018.

\section{Cours 6 - Le modèle de Harper-Hofstadter : l'effet Hall retrouvé}

Nous avons abordé dans ce dernier cours le problème qui est en fait à l'origine de la notion de bandes topologiques : l'effet Hall quantique. C'est en effet l'analyse du mouvement quantique à deux dimensions d'un ensemble de charges placées dans un champ magnétique qui a montré la quantification de grandeurs liées au transport, comme la conductivité de Hall. C'est également cette analyse qui a mis en avant l'importance des états de bord et qui a permis de dégager la notion de robustesse topologique en physique de la matière condensée. A priori, l'étude de l'effet Hall quantique ne nécessite pas de réseau sous-jacent. Toutefois, pour faire le lien avec les cours qui précèdent, nous nous sommes concentrés sur le cas où un potentiel périodique est également présent. La possibilité de traiter ce potentiel dans la limite des liaisons fortes permet alors de simplifier considérablement l'analyse : c'est le modèle de Harper-Hofstadter. Une fois ce modèle posé, nous avons étudié ses implémentations récentes (2015-2018) en physique atomique et en photonique. Nous avons montré comment elles ont permis la mesure explicite du nombre de Chern des bandes d'énergie. Nous avons également décrit comment les états de bord associés à cette topologie non triviale permettent de réaliser de nouveaux dispositifs, des lasers topologiques, qui exploitent la robustesse des bandes ainsi formées.

\section{Séminaire 1 - Photoionization dynamics on the attosecond time scale}

Anne L'Huillier (université de Lund, Suède), le 2 mai 2018

The interaction of atoms with intense laser radiation leads to the generation of high-order harmonics of the laser field. In the time domain, this corresponds to a train of pulses in the extreme ultraviolet range and with attosecond duration. The short pulse duration and broad bandwidth of attosecond pulses allow us to measure the phase and amplitude of electronic wave packets created by photoionization, using an interferometric technique. This gives us access to the temporal dynamics of the 
process that led to this wave-packet. The presentation has introduced the physics of high-order harmonic generation and attosecond pulses, and described applications concerning photoionization dynamics.

\section{Séminaire 2 - Building quantum systems from scratch}

Tilman Esslinger (ETH Zurich, Suisse), le 9 mai 2018

Cooling and manipulating atomic gases have opened up new avenues to explore fundamental concepts in quantum many-body physics. Synthetically created potentials and control of atom-atom interactions have made it possible to tailor the properties of experimental systems at a microscopic level. This led to the concept of quantum simulation - here a system capable of reproducing the physics of manybody Hamiltonians. One of the goals of this approach is to provide answers to open questions in the context of condensed matter physics. An equally important frontier is the construction of novel systems, which may at present not be realizable in solidstate or other systems. This path leads to new questions and surprises.

\section{Séminaire 3 - Entanglement creation and characterization in a trapped-ion quantum simulator}

Christian Roos (IQOQUI, Innsbruck, Académie des sciences d'Autriche), le 16 mai 2018

The seminar presented the generation and characterization of entangled states of a register of 20 individually controlled qubits, where each qubit is encoded into the electronic state of a trapped atomic ion. Entanglement is generated amongst the qubits during the out-of-equilibrium dynamics of an Ising-type Hamiltonian, engineered via laser fields. The main result of this study is that, during the dynamical evolution, all neighboring qubit pairs, triplets, most quadruplets, and some quintuplets simultaneously develop genuine multipartite entanglement.

\section{Séminaire 4 - Generating and manipulating single photons with semiconductor devices}

Pascale Senellart-Mardon (Centre pour les nanosciences et les nanotechnologies, CNRS, Marcoussis), le 23 mai 2018

Single-photons are key elements of many future quantum technologies, be it for the realization of large-scale quantum communication networks, for quantum simulation of chemical and physical processes, or for connecting quantum memories in a quantum computer. Scaling quantum technologies will thus require efficient, on-demand, sources of highly indistinguishable single-photons. The seminar is devoted to the recent realization of such devices made of quantum dots in electrically controlled cavity structures. The achieved device allows on-demand, bright and ultra-pure single photon generation, with a source 20 times brighter than any source of equal quality. This new generation of sources opens the way to a new level of complexity and scalability in optical quantum manipulation. 


\section{Séminaire 5 - La mission spatiale Microscope pour le test du principe d'équivalence}

Gilles Métris (Observatoire de la Côte d'Azur, Valbonne), le 30 mai 2018

$\mathrm{Au} \mathrm{XVII}^{\mathrm{e}}$ siècle, Galilée imagine une expérience où deux objets de nature différente tombent en même temps. Partant du fait que les deux corps touchent le sol au même moment, Galilée en déduit que, dans le vide, tous les corps tombent avec la même vitesse, quelles que soient leur masse ou leur composition. Cette universalité de la chute libre, ou encore l'équivalence entre la masse pesante et la masse inerte, sera érigée en principe, dit principe d'équivalence, par Albert Einstein qui en fera le fondement de la relativité générale. Le satellite Microscope a pour but de tester ce principe avec une précision cent fois meilleure que dans une expérience terrestre. Dans l'espace, il est en effet possible d'étudier le mouvement relatif des deux corps en réalisant une chute libre à l'abri des perturbations sismiques, et avec des mesures s'étalant sur plusieurs mois d'affilée. Le séminaire a été consacré à la présentation des premiers résultats de cette mission et à la description de la méthodologie utilisée.

\section{Séminaire 6 - Light manipulation in complex media: imaging and beyond}

Sylvain Gigan (Laboratoire Kastler Brossel, Paris), le 6 juin 2018

Light propagation in complex media is a highly complex and seemingly unpredictable process, which spans a wide range of situations from white paint, milk, fog, multimode fibers, and biological tissues. Yet, it is a coherent and deterministic process. Exploiting concepts inspired by astronomy, and thanks to the emergence of wave-control devices, such as liquid crystal displays and MEMS technology, a revolution has happened in the last decade: the possibility to control light and focus it through or inside scattering materials, well beyond what was conventionally thought possible. These concepts were illustrated through some examples from the seminar speaker's research. In particular, it was shown how the measurement of the transmission matrix of such medium allows focusing, imaging, and even arbitrary PSF generation through highly disordered media.

\section{COURS À L'EXTÉRIEUR - EXPLORING FLATLAND WITH COLD ATOMS}

Université de Trento, Italie, 12-13 décembre 2017 (2 cours)

Technion Institute, Haifa, Israel, 14-18 janvier 2018 (3 cours)

The physics of many-body systems strongly depends on their dimensionality. For example, in a two-dimensional world, most standard phase transitions towards an ordered state of matter would not occur because of the increased role of fluctuations. However, unconventional «topological» transitions can still take place in Flatland, and novel phases of matter also characterized by a non-trivial topology - e.g. Quantum Hall states - can emerge. The 2016 Physics Nobel prize was awarded to Kosterlitz, Thouless and Haldane for their pioneering work in this field. In this set of lectures, I described recent experimental investigations of Flatland physics performed with cold atomic gases. In particular, I presented the realization of uniform atomic gases confined in «square box » potentials, which has opened a number of perspectives in the physics of quantum fluids. These uniform systems are 
particularly relevant for the study of critical phenomena where one expects a divergence of correlation lengths. I also reviewed the recent progress in the formation of topological matter with cold atom setups, based on the interplay between light and matter and/or a periodic drive of the system.

\section{RECHERCHE}

La recherche de l'équipe de la chaire Atomes et rayonnement porte sur l'étude des gaz quantiques d'atomes refroidis à très basse température. Le comportement collectif de ces particules permet, d'une part, de simuler des phénomènes déjà connus pour les fluides d'électrons dans les solides, et, d'autre part, d'explorer de nouveaux régimes inatteignables avec des systèmes physiques « usuels ». On peut ainsi espérer obtenir avec ces gaz d'atomes froids des réponses expérimentales à des questions ouvertes en physique statistique et en physique de la matière condensée.

Au cours de cette année académique, notre recherche expérimentale a d'abord porté sur l'étude de l'ordre magnétique dans les gaz spineurs. En utilisant un montage centré sur le refroidissement d'atomes de sodium, nous avons montré que des domaines de spin pouvaient se former spontanément lors d'une transition de phase, en l'absence de toute force extérieure. Ces domaines, qui résultent uniquement de l'interaction antiferromagnétique entre atomes, conduisent à une dynamique du système particulièrement riche que nous avons caractérisée. Sur un autre montage expérimental utilisant des atomes de rubidium, nous avons mis en évidence l'existence d'ondes sonores dans un gaz bidimensionnel superfluide. Cette expérience constitue la première observation du second son dans un fluide quantique de dimension réduite et elle fournit des informations importantes sur la densité superfluide du gaz, une quantité conceptuellement importante, mais difficile à mesurer directement. Notre étude de l'extension de ce mode sonore au cas non-superfluide a conduit à des résultats inattendus, qui ont fait l'objet de nombreuses discussions notamment avec le groupe de théoriciens de l'université de Trento. Un troisième montage de notre équipe utilise des atomes d'ytterbium placés dans un réseau optique. Nous l'avons mis à profit pour étudier la perte de cohérence spatiale d'un gaz quantique en interaction forte. Cette décohérence est initiée par l'addition d'un champ laser provoquant une émission spontanée contrôlée. Nous avons mis en évidence une décroissance anormalement lente de la cohérence et nous l'avons attribuée à l'effet des interactions entre atomes qui fournissent ainsi une protection effective. Dans l'espace des vitesses, cela se traduit par une diffusion moins rapide que la diffusion en vitesses bien connue dans le contexte du refroidissement laser. Notre quatrième et dernier montage expérimental utilise des atomes de dysprosium. Nous avons mis à profit la structure de niveau très particulière de cet atome, qui possède un grand moment cinétique dans son état fondamental, pour produire des états de spin au comportement fortement non classique. Ces états sont une superposition quantique de deux états de magnétisation opposée, et peuvent donc être vus comme une réalisation d'un (petit) chat de Schrödinger. Nous avons exploré l'intérêt métrologique de ces états, par exemple pour la mesure de faibles champs magnétiques.

En parallèle avec cette recherche expérimentale, nous poursuivons également une activité théorique au sein de l'équipe de chaire. Cette activité s'est notamment traduite par la rédaction d'un «gros » article de revue sur la réalisation de bandes topologiques dans les gaz d'atomes froids, en collaboration avec des chercheurs de 
l'université de Cambridge (Royaume-Uni) et du Maryland (États-Unis). Une autre étude, menée avec des collègues italiens, a porté sur le comportement d'un gaz d'atomes en interaction, placé sur un réseau de type échelle. Il s'est agi d'identifier les différentes phases susceptibles d'apparaître en présence d'un champ de jauge, notamment la phase Meissner et la phase vortex. Cette étude pourra servir de base à une future expérience menée dans notre groupe.

\section{PuBLiCATIONS}

Dareau A., Levy E., Bosch Aguilera M., Bouganne R., AkKermans E., Gerbier F. et BEUGNON J., « Revealing the topology of quasicrystals with a diffraction experiment », Physical Review Letters, vol. 119, no 21, 2017, 215304, DOI : 10.1103/PhysRevLett.119.215304 [arXiv :1607.00901].

Saint-Jalm R., Aidelsburger M., Ville J.L., Corman L., Hadzibabic Z., Delande D., NASCIMBEne S., CHERroret N., DALIBARD J. et BEUGNON J., « Resonant-light diffusion in a disordered atomic layer », Physical Review A, vol. 97, n 6, 2018, 061801, DOI : 10.1103/ PhysRevA.97.061801 [arXiv : 1802.04018].

Bosch Aguilera M., Bouganne R., Dareau A., Scholl M., Beaufils Q., Beugnon J. et GERBIER F., « Non-linear relaxation of interacting bosons coherently driven on a narrow optical transition », EPL : Europhysics Letters, vol. 123, no 4, 2018, 40004, DOI : 10.1209/ 0295-5075/123/40004 [arXiv: 1806.07210].

Calvanese Strinati M.C., Gerbier F. et Mazza L., « Spin-gap spectroscopy in a bosonic flux ladder », New Journal of Physics, vol. 20, n 1, 2018, 015004, DOI : 10.1088/1367-2630/ aa9ca2 [arXiv: 1708.02832].

Ville J.L., SAint-Jalm R., Le Cerf É., Aidelsburger M., NAScimbÈne S., Dalibard J. et BEUGNON J., «Sound propagation in a uniform superfluid two-dimensional Bose gas », Physical Review Letters, vol. 121, n $14,2018,145301$, DOI : 10.1103/PhysRevLett.121.145301 [arXiv: 1804.04037].

Cooper N.R., Dalibard J. et SpIElman I.B., « Topological bands for ultracold atoms », 2018, http://arxiv.org/abs/1803.00249.

Chalopin T., Makhalov V., Bouazza C., Evrard A., BARKer A., LePers M., Wyart J.-F., Dulieu O., Dalibard J., LOPES R. et NASCimbene S., « Anisotropic light-shift and magicpolarization of the intercombination line of Dysprosium atoms in a far-detuned dipole trap », Physical Review A, vol. 98, no 4, 2018, 040502, DOI : 10.1103/PhysRevA.98.040502 [arXiv: 1805.06878].

Chalopin T., Bouazza C., Evrard A., Makhalov V., Dreon D., Dalibard J., SIDORENKOV L.A. et NASCIMBENE S., "Quantum-enhanced sensing using non-classical spin states of a highly magnetic atom », Nature Communications, vol. 9, $\mathrm{n}^{\circ}$ 1, 2018, 4955, DOI : 10.1038/s41467-018-07433-1 [arXiv: 1806.05495].

JimÉNEZ-García K., InVERnizzi A., Evrard B., Frapolli C., DAlibard J. et Gerbier F., «Spontaneous formation and relaxation of spin domains in antiferromagnetic spin-1 quasicondensates », 2018, http://arxiv.org/abs/1808.01015. 